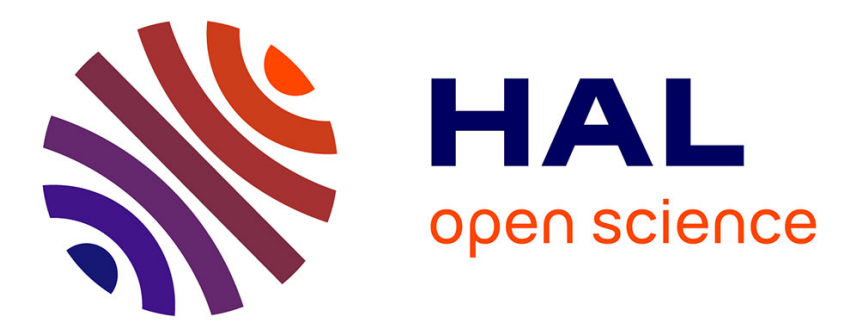

\title{
Effect of regularization of Schmid law on self-consistent estimates for rate-independent plasticity of polycrystals
}

Kengo Yoshida, Renald Brenner, Brigitte Bacroix, Salima Bouvier

\section{To cite this version:}

Kengo Yoshida, Renald Brenner, Brigitte Bacroix, Salima Bouvier. Effect of regularization of Schmid law on self-consistent estimates for rate-independent plasticity of polycrystals. European Journal of Mechanics - A/Solids, 2009, 28 (5), pp.905. 10.1016/j.euromechsol.2009.05.001 . hal-00503302

\section{HAL Id: hal-00503302 \\ https://hal.science/hal-00503302}

Submitted on 19 Jul 2010

HAL is a multi-disciplinary open access archive for the deposit and dissemination of scientific research documents, whether they are published or not. The documents may come from teaching and research institutions in France or abroad, or from public or private research centers.
L'archive ouverte pluridisciplinaire HAL, est destinée au dépôt et à la diffusion de documents scientifiques de niveau recherche, publiés ou non, émanant des établissements d'enseignement et de recherche français ou étrangers, des laboratoires publics ou privés. 


\section{Accepted Manuscript}

Title: Effect of regularization of Schmid law on self-consistent estimates for rateindependent plasticity of polycrystals

Authors: Kengo Yoshida, Renald Brenner, Brigitte Bacroix, Salima Bouvier

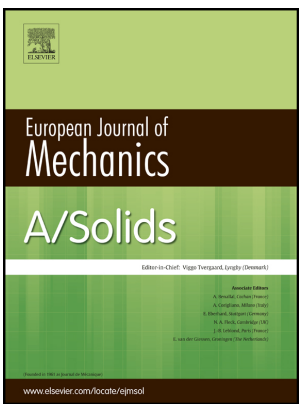

PII:

S0997-7538(09)00061-8

DOI:

10.1016/j.euromechsol.2009.05.001

Reference: EJMSOL 2525

To appear in: European Journal of Mechanics / A Solids

Received Date: 30 January 2009

Revised Date: 28 April 2009

Accepted Date: 1 May 2009

Please cite this article as: Yoshida, K., Brenner, R., Bacroix, B., Bouvier, S. Effect of regularization of Schmid law on self-consistent estimates for rate-independent plasticity of polycrystals, European Journal of Mechanics / A Solids (2009), doi: 10.1016/j.euromechsol.2009.05.001

This is a PDF file of an unedited manuscript that has been accepted for publication. As a service to our customers we are providing this early version of the manuscript. The manuscript will undergo copyediting, typesetting, and review of the resulting proof before it is published in its final form. Please note that during the production process errors may be discovered which could affect the content, and all legal disclaimers that apply to the journal pertain. 


\section{Effect of regularization of Schmid law on self-consistent estimates for rate-independent plasticity of polycrystals}

6 a LPMTM, CNRS, University Paris 13, 99 Avenue Jean-Baptiste Clement, 93430

7 Villetaneuse, France

8 b Forming Technologies R\&D Center, Steel Research Laboratories, Nippon Steel

9 Corporation, 20-1 Shintomi, Futtsu, Chiba 293-8511, Japan

11 *Corresponding author

12 Tel.: +331494034 67; Fax: +33149403938.

13 E-mail address: yoshida.kengo1@nsc.co.jp (K. Yoshida).

\section{Keywords}

29 Self-consistent model, rate-independent elastoplasticity, polycrystal, nonlinear

30 homogenization 


\section{Introduction}

32 Homogenization methods are efficient approaches to estimate the mechanical properties of

33 heterogeneous materials. In the case of polycrystalline media, theoretical results have shown

34 that the linear elastic self-consistent model (Hershey, 1954; Kröner, 1958) is well appropriated

35 to describe their effective properties (Kröner, 1978). This has been assessed by comparing the

36 effective behavior and the local fields with results delivered by full-field computations on

37 various polycrystalline microstructures (see, for instance, Lebensohn et al., 2004; Castelnau et

38 al., 2006; Brenner et al., submitted).

40 The extension of the self-consistent model to rate-independent elastoplastic behavior has first

41 been proposed by Budiansky et al. (1960) and Kröner (1961) who investigated the early stage

42 of plasticity by considering plastic grains interacting with an elastic matrix. To describe

43 entirely the elastoplastic response of polycrystals, a seminal work was further introduced by

44 Hill (1965) and Hutchinson (1970) who proposed an incremental approach based on the linear

45 rate-form of local constitutive equations which rely on the Schmid law. More recently, an

46 affine approach has been proposed by Masson et al. (2000) who showed, for a non-hardening

47 f.c.c. polycrystal, that it leads to a lower steady-state effective yield stress than the 48 incremental model.

In the related context of power-law viscoplastic f.c.c. polycrystals, various extensions of the

51 self-consistent model, including the incremental (Hutchinson, 1976) and affine (Masson et al,

52 2000) approaches, have been compared to rigorous nonlinear bounds obtained with the

53 variational procedure of Ponte Castañeda (1991). Nebozhyn et al. (2001) have shown that the

54 incremental model violates the variational self-consistent estimate on the effective yield stress,

55 which is an upper bound for all self-consistnet estimates, and tends to the Taylor bound in the

56 rate-independent limit. By contrast, the affine model respects the available bounds, for this

57 specific class of polycrystals. In view of these results, Hill's incremental model (1965) could

58 be expected to present similar shortcomings in the context of rate-independent elastoplasticity.

59 However, a few works (Hutchinson, 1970 and Takahashi, 1988) show that the incremental

60 model estimates softer response than the Taylor bound for elastoplasticity, which contradicts 
61 the above expectation. The link between the rate-independent limit of viscoplasticity and

62 rate-independent elastoplasticity remains an open question.

64 In the present work, we analyze the influence of the local elastoplastic behavior on the

65 incremental self-consistent estimate of the polycrystal response. For that goal, two 66 rate-independent crystal plasticity models have been chosen. On one hand, use is made of the 67 Standard Schmid Law (SSL) in which plastic slip is considered to occur on a slip system 68 when the resolved shear stress reaches a critical value. This constitutive law leads multi-yield 69 functions and the determination of the slip rates is an inverse problem of the constraint 70 conditions. The constraints can be linearly dependent and result in the non-uniqueness of the 71 set of active slip systems when the hardening matrix is not positive definite (Hill, 1966). This 72 has led to various proposals for the hardening description (see, for instance, Franciosi and 73 Zaoui, 1991; Bassani, 1994) and for the technique to identify the set of slip systems (Anand 74 and Kothari, 1996; Miehe and Schröder, 2001; Busso and Cailletaud, 2005). A simple and 75 often used method to circumvent this difficulty is to forsake the rate-independent formulation 76 and adopt a power-law type rate-dependent viscoplastic formulation (see, for instance, Asaro 77 and Needleman, 1985). On the other hand, Regularized Schmid Law (RSL) has been 78 proposed to overcome this problem within the framework of the rate-independent plasticity 79 (see, Gambin, 1992; Darrieulat and Piot, 1996; Franz et al. 2009). The advantage of the 80 regularization is that it does not involve the problem of linear dependence. It has been used in 81 the homogenization context with the Taylor model (Darrieulat and Piot, 1996, Kowalczyk and 82 Gambin, 2004), the Transformation Field Analysis (Franciosi and Berbenni, 2007) and, 83 recently, the self-consistent model (Franz et al. 2009). In section 2, two crystal-plasticity 84 models (SSL and RSL) are presented and the corresponding tangent moduli in the rate-form 85 constitutive relations are compared for single and double slip cases in the single crystal 86 context. These crystal plasticity laws are then introduced in the Taylor and incremental 87 self-consistent models and the mechanical responses of two different non-hardening f.c.c. 88 polycrystals are estimated (Sections 3 and 4). The significance of the description of 89 constitutive law, especially the instantaneous tangent moduli, on the self-consistent estimates 90 is discussed in details in section 5. 


\section{Rate-independent crystal plasticity models}

93 2.1. Framework of crystal plasticity model

94 In this study, we confine attention to small strain conditions. The strain rate is given by the 95 symmetry part of $\partial \mathbf{v} / \partial \mathbf{x}$, where $\mathbf{v}$ and $\mathbf{x}$ are velocity and position, respectively. We 96 consider additive decomposition of strain rate into elastic and plastic parts.

$$
\dot{\boldsymbol{\varepsilon}}=\dot{\boldsymbol{\varepsilon}}^{\mathrm{e}}+\dot{\boldsymbol{\varepsilon}}^{\mathrm{p}}
$$

98 Elastic relation is given by Hooke's law.

$$
\dot{\boldsymbol{\sigma}}=\mathbf{C}^{\mathrm{e}}: \dot{\boldsymbol{\varepsilon}}^{\mathrm{e}}=\mathbf{C}^{\mathrm{e}}:\left(\dot{\boldsymbol{\varepsilon}}-\dot{\boldsymbol{\varepsilon}}^{\mathrm{p}}\right)
$$

100 where $\mathbf{C}^{\mathrm{e}}$ is the forth-order elastic moduli tensor.

Crystallographic slips are considered to be the source for the plastic deformation, and the plastic strain rate takes the form

$$
\dot{\boldsymbol{\varepsilon}}^{\mathrm{p}}=\sum_{\alpha} \dot{\gamma}^{(\alpha)} \mathbf{p}^{(\alpha)}
$$

where $\dot{\gamma}^{(\alpha)}, \mathbf{s}^{(\alpha)}$ and $\mathbf{m}^{(\alpha)}$ are the slip rate, the slip direction and the slip plane normal for

107 the $\alpha$ th slip system, respectively. The resolved shear stress for the $\alpha$ th slip system, $\tau^{(\alpha)}$, is 108 written as

$$
\mathbf{p}^{(\alpha)}:=\frac{1}{2}\left(\mathbf{s}^{(\alpha)} \otimes \mathbf{m}^{(\alpha)}+\mathbf{m}^{(\alpha)} \otimes \mathbf{s}^{(\alpha)}\right),
$$

110 where $\boldsymbol{\sigma}$ is true stress. From Equations (3) and (5), a resolved shear stress rate is given as

$$
\dot{\tau}^{(\alpha)}=\mathbf{p}^{(\alpha)}: \mathbf{C}^{\mathrm{e}}: \dot{\boldsymbol{\varepsilon}}-\sum_{\beta} \dot{\gamma}^{(\beta)} \mathbf{p}^{(\alpha)}: \mathbf{C}^{\mathrm{e}}: \mathbf{p}^{(\beta)}
$$

112 where $\mathbf{s}^{(\alpha)}$ and $\mathbf{m}^{(\alpha)}$ are assumed to be constant. Slip resistance for the $\alpha$ th slip system is 113 denoted by $g^{(\alpha)}$, and its evolution is governed by 


$$
\dot{\boldsymbol{g}}^{(\alpha)}=\sum_{\beta} h^{\alpha \beta}\left|\dot{\gamma}^{(\beta)}\right|
$$

115 where $h^{\alpha \beta}$ denotes hardening moduli.

\subsection{Standard Schmid law}

118 For a crystal plasticity model based on the standard Schmid law, the local yield function, $f$, 119 is written as

$$
f=\sup _{\alpha=1, \cdots, N^{\mathrm{s}}} f^{(\alpha)}=0
$$

121 with

$$
f^{(\alpha)}=\left|\tau^{(\alpha)}\right|-g^{(\alpha)}=0,
$$

123 where $N^{s}$ is the number of slip systems and $f^{(\alpha)}$ is a yield function for each slip system.

124 The plastic strain rate is given by

125

$$
\dot{\boldsymbol{\varepsilon}}^{\mathrm{p}}=\sum_{\alpha} \operatorname{sgn}\left(\tau^{(\alpha)}\right) \dot{\gamma}^{(\alpha)} \mathbf{p}^{(\alpha)},
$$

126 where slip rates, $\dot{\gamma}^{(\alpha)}$, are assumed to be positive. It is worth noting that the above equation

127 can be derived from the normality rule if the yield function for each slip system is considered as a potential: $\quad \dot{\boldsymbol{\varepsilon}}^{\mathrm{p}}=\sum_{\alpha} \dot{\gamma}^{(\alpha)}\left(\partial f^{(\alpha)} / \partial \boldsymbol{\sigma}\right)$.

130 Based on $N^{s}$ independent yield functions (Equation (9)) potentially active and inactive slip 131 systems are classified as

$$
\dot{\gamma}^{(\alpha)} \geq 0, \text { for } f^{(\alpha)}=0 \text { and } \dot{f}^{(\alpha)}=0 \text {, }
$$

134 From the consistency condition of the yield functions, the slip rates, $\dot{\gamma}^{(\alpha)}$, on the active slip 135 systems are determined as

$$
\dot{f}^{(\alpha)}=R^{\alpha}-\sum_{\beta} X^{\alpha \beta} \dot{\gamma}^{(\beta)}=0
$$




$$
\dot{\gamma}^{(\alpha)}=\sum_{\beta} Y^{\alpha \beta} R^{(\beta)},
$$

138 where

$$
X^{\alpha \beta}:=h^{\alpha \beta}+\operatorname{sgn}\left(\tau^{(\alpha)}\right) \operatorname{sgn}\left(\tau^{(\beta)}\right) \mathbf{p}^{(\alpha)}: \mathbf{C}^{\mathrm{e}}: \mathbf{p}^{(\beta)},
$$

$$
\left[Y^{\alpha \beta}\right]:=\left[X^{\alpha \beta}\right]^{-1},
$$

142 where $(\bullet)^{-1}$ denotes the inverse. Under a particular hardening rule, $h^{\alpha \beta}, X^{\alpha \beta}$ may become

143 singular, and this results in a possible non-uniquess of the set of the active slip systems for a 144 given deformation mode. But for perfect plasticity, $h^{\alpha \beta}=0$, it is always possible to choose at 145 least one set of linearly independent slip systems from the potentially active slip systems such 146 that $X^{\alpha \beta}$ is non-singular and Equations (11) are satisfied (Hutchinson, 1970). If there are 147 greater than five linearly independent systems, five slip systems are selected in the 148 computations ${ }^{1}$.

150 Using equations (2), (10) and (13), we finally obtain the rate-form of the constitutive

151 equation, $\dot{\boldsymbol{\sigma}}=\mathbf{L}: \dot{\boldsymbol{\varepsilon}}$, in term of the elastoplastic tangent moduli,

$$
\mathbf{L}:=\mathbf{C}^{\mathrm{e}}-\sum_{\alpha}\left\{\left(\operatorname{sgn}\left(\tau^{(\alpha)}\right) \mathbf{C}^{\mathrm{e}}: \mathbf{p}^{(\alpha)}\right) \otimes \sum_{\beta}\left(\operatorname{sgn}\left(\tau^{(\beta)}\right) Y^{\alpha \beta} \mathbf{p}^{(\beta)}: \mathbf{C}^{\mathrm{e}}\right)\right\} .
$$

\subsection{Regularized Schmid law}

155 In order to avoid the possible singularity of $X^{\alpha \beta}$ in the Schmid law, a regularized Schmid 156 law for rate-independent crystal plasticity was proposed (Gambin, 1992). The following

\footnotetext{
${ }^{1}$ For comparison purpose we use the same approach as Hutchinson's (1970). In a preliminary study, different criteria have been used for the selection of the five active slip systems, and it has been confirmed that the choice of the criterion does not have any influence on the estimation.
} 
157 regularized yield function is introduced.

158

$$
f=\left\{\sum_{\alpha}\left(\frac{\tau^{(\alpha)}}{g^{(\alpha)}}\right)^{2 N}\right\}^{\frac{1}{2 N}}-1=0,
$$

159 where $N$ is an integer parameter so that the exponent always becomes an even number. The 160 plastic strain rate is assumed to be given by

$$
\dot{\boldsymbol{\varepsilon}}^{\mathrm{p}}=\sum_{\alpha} \dot{\gamma}^{(\alpha)} \mathbf{p}^{(\alpha)}
$$

162 and follows, on the other hand, from the normality rule associated with the yield function 163 (16),

$164 \quad \dot{\boldsymbol{\varepsilon}}^{\mathrm{p}}=\dot{\Lambda} \frac{\partial f}{\partial \boldsymbol{\sigma}}$.

165 From these two expressions of the plastic strain rate, the slip rate on a given slip system reads

$$
\dot{\gamma}^{(\alpha)}=\frac{\dot{\lambda}}{g^{(\alpha)}}\left(\frac{\tau^{(\alpha)}}{g^{(\alpha)}}\right)^{2 N-1} \quad \text { with } \quad \dot{\lambda}=\dot{\Lambda}\left\{\sum_{\alpha}\left(\frac{\tau^{(\alpha)}}{g^{(\alpha)}}\right)^{2 N}\right\}^{\frac{1}{2 N}-1},
$$

where $\dot{\lambda}$ is a positive plastic multiplier. By contrast to the SSL, slip rates for all the slip systems derived from a unique plastic multiplier. Therefore, there is no need to classify active and inactive slip systems among the set of slip systems. Equation (19) is similar to the one describing vicsoplastic crystal (e.g. Asaro and Needleman, 1985) in which a reference slip

171 rate is introduced instead of a plastic multiplier.

173 Loading-unloading conditions are written as

$$
\dot{\lambda} \geq 0, \text { for } f=0 \text { and } \dot{f}=0 \text {, }
$$

176 From the consistency condition of the yield function, the plastic multiplier, $\dot{\lambda}$, is determined.

$$
\dot{f}=\mathbf{G}: \mathbf{C}^{\mathrm{e}}: \dot{\boldsymbol{\varepsilon}}-\dot{\lambda}\left(\mathbf{G}: \mathbf{C}^{\mathrm{e}}: \mathbf{G}+H\right)=0
$$




$$
\dot{\lambda}=\frac{\mathbf{G}: \mathbf{C}^{\mathrm{e}}: \dot{\boldsymbol{\varepsilon}}}{\mathbf{G}: \mathbf{C}^{\mathrm{e}}: \mathbf{G}+H}
$$

179 where

$$
\begin{aligned}
\mathbf{G} & :=\sum_{\alpha}\left\{\left(\frac{\tau^{(\alpha)}}{g^{(\alpha)}}\right)^{2 N-1} \frac{\mathbf{p}^{(\alpha)}}{g^{(\alpha)}}\right\}, \\
H & :=\sum_{\alpha}\left[\left(\frac{\tau^{(\alpha)}}{g^{(\alpha)}}\right)^{2 N} \frac{1}{g^{(\alpha)}} \sum_{\beta}\left\{\frac{h_{\alpha \beta}}{g^{(\beta)}}\left(\frac{\left|\tau^{(\beta)}\right|}{g^{(\beta)}}\right)^{2 N-1}\right\}\right] .
\end{aligned}
$$

182 In the derivation of $\dot{\lambda}$, any inverse of a matrix is not required. Namely, the difficulty related 183 to the linearly dependence in the Schmid law is avoided.

185 We finally obtain the rate-form constitutive equation for a single crystal, $\dot{\boldsymbol{\sigma}}=\mathbf{L}: \dot{\boldsymbol{\varepsilon}}$, in terms of 186 the elastoplastic tangent moduli,

$$
\mathbf{L}:=\mathbf{C}^{\mathrm{e}}-\frac{\left(\mathbf{C}^{\mathrm{e}}: \mathbf{G}\right) \otimes\left(\mathbf{G}: \mathbf{C}^{\mathrm{e}}\right)}{\mathbf{G}: \mathbf{C}^{\mathrm{e}}: \mathbf{G}+H}
$$

\subsection{Comparison of Schmid and regularized Schmid laws}

190 In this subsection, the standard Schmid and regularized Schmid laws are compared in terms of yield locus, active slip systems and tangent moduli. The SSL gives multi-plane yield locus which possesses corners, while the RSL gives a smooth yield locus with rounded corners. The curvature of the rounded corner for the RSL increases with the exponent $N$, and when $N$ is large enough, the smooth yield locus is in good agreement with the multi-plane SSL's one (Darrieulat and Piot, 1996; Gambin and Barlat, 1997). Besides, although all the slip systems are active if the yield condition is satisfied, finite slips take place only on the slip systems where $\tau^{(\alpha)} \approx g^{(\alpha)}$ when the exponent $N$ is large, while slip rates for the other slip systems, where $\tau^{(\alpha)}<g^{(\alpha)}$, are close to zero (Equation (19)). This is similar to the Schmid law, for which slip system activates when $\tau^{(\alpha)}=g^{(\alpha)}$. Therefore, the yield locus and active slip systems corresponding to the Schmid law are correctly described by the regularized Schmid law when $N \rightarrow+\infty$. On the contrary, the tangent moduli given in Equation (15) and (24) are not identical a priori. To be more specific, the tangent moduli given by the two laws are compared for some special cases. 
(i) Single slip case

206 Let us consider a crystal that possesses a single slip system. The exponent in the regularized

207 Schmid law is taken to $N=+\infty$. In this case, the yield function (16) reduces to Equation (9).

208 It is assumed that $\tau^{(1)}=g^{(1)}$ and $\dot{\tau}^{(1)}=\dot{g}^{(1)}$ for the slip system. This situation results in the 209 activation of the slip system for both SSL and RSL. Then the tangent moduli is written as

$$
\mathbf{L}=\mathbf{C}^{\mathrm{e}}-\frac{\left(\mathbf{C}^{\mathrm{e}}: \mathbf{p}^{(1)}\right) \otimes\left(\mathbf{p}^{(1)}: \mathbf{C}^{\mathrm{e}}\right)}{\mathbf{p}^{(1)}: \mathbf{C}^{\mathrm{e}}: \mathbf{p}^{(1)}+h^{11}}
$$

The tangent moduli (15) and (24) are reduced to the same equation.

\section{(ii) Double slip case}

214 Next, let us consider a crystal that possesses two slip systems. It is assumed that $\tau^{(1)}=g^{(1)}$, $\tau^{(2)}=g^{(2)}$ and $\dot{\tau}^{(1)}=\dot{g}^{(1)}, \dot{\tau}^{(2)}=\dot{g}^{(2)}$. For both the Schmid and regularized Schmid laws, the yield criteria are satisfied and slips occur for both the first and second slip systems. These situations are similar to that for the single slip case. In this case, the tangent moduli obtained with the Schmid law (Equation(15)) reduces to

220 where

$$
\mathbf{L}=\mathbf{C}^{\mathrm{e}}-\mathbf{C}^{\mathrm{e}}:\left\{\mathbf{p}^{(1)} \otimes\left(Y^{11} \mathbf{p}^{(1)}+Y^{12} \mathbf{p}^{(2)}\right)+\mathbf{p}^{(2)} \otimes\left(Y^{21} \mathbf{p}^{(1)}+Y^{22} \mathbf{p}^{(2)}\right)\right\}: \mathbf{C}^{\mathrm{e}},
$$

\section{where}

$$
\left[\begin{array}{ll}
Y^{11} & Y^{12} \\
Y^{21} & Y^{22}
\end{array}\right]=\left[\begin{array}{cc}
h^{11}+\mathbf{p}^{(1)}: \mathbf{C}^{\mathrm{e}}: \mathbf{p}^{(1)} & h^{12}+\mathbf{p}^{(1)}: \mathbf{C}^{\mathrm{e}}: \mathbf{p}^{(2)} \\
h^{21}+\mathbf{p}^{(2)}: \mathbf{C}^{\mathrm{e}}: \mathbf{p}^{(1)} & h^{22}+\mathbf{p}^{(2)}: \mathbf{C}^{\mathrm{e}}: \mathbf{p}^{(2)}
\end{array}\right]^{-1},
$$

and $g^{(1)}=g^{(2)}$ is assumed for simplicity.

224 With the regularized Schmid law, the tangent moduli (Equation(24)) gives

$$
\mathbf{L}=\mathbf{C}^{\mathrm{e}}-\frac{\mathbf{C}^{\mathrm{e}}:\left(\mathbf{p}^{(1)}+\mathbf{p}^{(2)}\right) \otimes\left(\mathbf{p}^{(1)}+\mathbf{p}^{(2)}\right): \mathbf{C}^{\mathrm{e}}}{\left(\mathbf{p}^{(1)}+\mathbf{p}^{(2)}\right): \mathbf{C}^{\mathrm{e}}:\left(\mathbf{p}^{(1)}+\mathbf{p}^{(2)}\right)+h_{11}+h_{12}+h_{21}+h_{22}} .
$$

226 For the double slip case, the two crystal plasticity laws do not give the identical expression of tangent moduli. For the Schmid law, interaction of $\mathbf{p}^{(\alpha)}$ for each slip system appears with different coefficient, $Y^{\alpha \beta}$, while interaction of the summation of $\mathbf{p}^{(\alpha)}$ emerges for the regularized Schmid law. 
231 The tangent moduli given by the two laws are calculated for a special case shown in Figure 1

232 (a). Two slip systems are arranged symmetrically about the $x_{1}$ axis and slip directions and 233 slip plane normals are in the $x_{1}-x_{2}$ plane, that is, $234 \quad \mathbf{s}^{(1)}=(\cos \phi, \sin \phi, 0) \quad \mathbf{m}^{(1)}=(\sin \phi,-\cos \phi, 0) \quad, \quad \mathbf{s}^{(2)}=(\cos \phi,-\sin \phi, 0) \quad$ and

$235 \mathbf{m}^{(2)}=(\sin \phi, \cos \phi, 0)$. For simplicity, isotropic elasticity and non-hardening is assumed. 236 Then, analytical expression of tangent moduli are written as

$$
L_{1111}=L_{2222}=L_{1122}=\lambda+\mu
$$

where $\lambda$ and $\mu$ are Lamé constants. The difference of tangent modulus is appeared for $L_{1212}$ component.

In the derivation of the analytical expression of the tangent moduli $N=+\infty$ is assumed,

244 however, a finite value of $N$ is required in actual computation. In order to check the sensitivity of $N$, the same problem is solved numerically with $N=500$. Parameters are set to $\phi=15^{\circ}, E / \tau_{0}=1000, v=1 / 3$ and $h^{\alpha \beta}=0$, where $E, v$ and $\tau_{0}$ are the Young's

248 the $x_{1}$ direction is applied. Figure 1(b) shows the tangent moduli normalized by $E$ as a 249 function of the tensile strain. Tangent moduli given by the two crystal plasticity laws coincide 250 except for the $L_{1212}$ component, and $L_{1212}$ for the Schmid law decrease to zero when the 251 yield condition is satisfied, whereas that for the regularized Schmid law remains constant. The 252 tangent moduli computed with $N=500$ coincide with those obtained analytically with $253 N=+\infty$. Figure 1(c) shows the good agreement between SSL and RSL tensile flow stresses. 254 Thus, the yield stress and active slip systems are the same for both crystal plasticity laws 255 whereas the shear tangent moduli are different.

257 Following these comparisons at the single crystal level, the question which arises is the 
258 influence, if any, of the different instantaneous local tangent moduli on the polycrystal

259 response. To tackle this problem, use will be made of the Taylor and self-consistent models

260 which are briefly described in the next section.

\section{3. Homogenization estimates}

\section{3.1. Self-consistent model}

264 In this study, we mainly focus on the role of the crystal plasticity law on the local and 265 effective response for the elastoplastic polycrystalline aggregate. Consequently, the Hill's 266 (1965) incremental self-consistent model, which is up to now the most widely used model for 267 elastoplastic polycrystal, is adopted. Polycrystal is considered to consist of ellipsoidal phases 268 which compose grains orientating at the same direction. In the self-consistent model, an 269 ellipsoidal phase is assumed to be embedded in an infinite linear comparison homogenous 270 medium which has a unique tangent moduli corresponding to the effective tangent ones. The 271 phase average stress and strain rates can be related to the macroscopic ones through the 272 localization tensors, $\mathbf{A}^{r}$ and $\mathbf{B}^{r}$,

$$
<\dot{\boldsymbol{\varepsilon}}>^{r}=\mathbf{A}^{r}: \dot{\overline{\boldsymbol{\varepsilon}}}, \quad<\dot{\boldsymbol{\sigma}}>^{r}=\mathbf{B}^{r}: \dot{\overline{\boldsymbol{\sigma}}},
$$

274 where the superscript $r$ refers to the quantity for the $r$ th phase, a bar indicates a 275 macroscopic value and $<\bullet>$ stands for a volume average. The rate-form constitutive 276 equation is used to represent linear relationship between the stress and strain rates. The 277 constitutive equations of a phase and effective medium are respectively denoted as

with

$$
<\dot{\boldsymbol{\sigma}}>^{r}=\mathbf{L}^{r}:<\dot{\boldsymbol{\varepsilon}}>^{r}
$$

$$
\dot{\overline{\boldsymbol{\sigma}}}=\tilde{\mathbf{L}}: \dot{\overline{\boldsymbol{\varepsilon}}}
$$

282 where $\mathbf{L}^{r}$ and $\tilde{\mathbf{L}}$ are the tangent moduli of a phase and effective medium, respectively. $\mathbf{L}^{r}$ 283 is assumed to be homogeneous within a phase and can be calculated by Equation (15) or (24) 284 replacing the local quantities with the phase average ones, for instance, a resolved shear stress 285 $<\tau^{(\alpha)}>^{r}=\mathbf{p}^{(\alpha)}:<\boldsymbol{\sigma}>^{r}$ is used instead of $\tau^{(\alpha)}=\mathbf{p}^{(\alpha)}: \boldsymbol{\sigma}$. The strain localization tensor is given 
by

$$
\mathbf{A}^{r}=\left\{\mathbf{I}+\mathbf{P}:\left(\mathbf{L}^{r}-\tilde{\mathbf{L}}\right)\right\}^{-1}
$$

288 where $\mathbf{I}$ is fourth-order identity tensor and $\mathbf{P}$ is so called Hill's tensor described as

$$
\mathbf{P}=\int_{\Omega} \Gamma \mathrm{d} V,
$$

290 where

$$
\Gamma_{i j k l}:=\frac{1}{4}\left(K_{i k}^{-1} \xi_{j} \xi_{l}+K_{j k}^{-1} \xi_{i} \xi_{l}+K_{i l}^{-1} \xi_{j} \xi_{k}+K_{j l}^{-1} \xi_{i} \xi_{k}\right)
$$

$$
\mathbf{K}:=\xi \cdot \tilde{\mathbf{L}} \cdot \xi
$$

where $\Omega$ is the domain within a phase and $\xi$ is a position vector on the surface of inclusion (see, for instance, Mura, 1982). This set of relations defines a nonlinear systems for the average strain rates per phase, $\left\langle\dot{\boldsymbol{\varepsilon}}>^{r}\right.$, which can be solved using a fixed-point iterative

\subsection{Taylor model}

In the Taylor model, $\mathbf{A}^{r}=\mathbf{I}$ is assumed, that is, the local strain field is taken to be identical to the macroscopic one,

$$
\dot{\boldsymbol{\varepsilon}}=<\dot{\boldsymbol{\varepsilon}}>^{r}=\dot{\overline{\boldsymbol{\varepsilon}}}
$$

302 Then, the effective tangent moduli in the rate-form of constitutive equation, $\dot{\overline{\boldsymbol{\sigma}}}=\tilde{\mathbf{L}}: \dot{\overline{\boldsymbol{\varepsilon}}}$, are 303 given by

$$
\tilde{\mathbf{L}}=<\mathbf{L}>\text {. }
$$

305 Since the local strain field is known a priori, iterative computation is not needed for the Taylor 306 model.

308 In the next section, the Taylor and incremental self-consistent models described above are 309 used in conjunction with SSL and RSL for estimating the mechanical behavior of f.c.c. polycrystals at both local and overall scales. 


\section{Results}

\subsection{Uniaxial tension for non-hardening f.c.c. polycrystalline aggregate}

314 Macroscopically isotropic polycrystal is considered. Its microstructure is represented by 216

315 crystalline phases with same volume fractions. Besides, a spherical shape is assumed for the

316 each phase. A non-hardening ( $h^{\alpha \beta}=0$ ) f.c.c. crystal with 12 slip systems, $\{111\}<110>$, is

317 assumed for each phase, and slip resistances are set as $g^{(\alpha)}=\tau_{0}$, where $\tau_{0}$ is a critical

318 resolved shear stress. For simplicity isotropic elasticity with $E / \tau_{0}=1000$ and $v=1 / 3$ is

319 considered. The exponent in the regularized Schmid laws is $N=500$. (The effect of the

320 exponent, $N$, on homogenization estimates is discussed in Appendix.) Mechanical response

321 of the polycrystal subjected to the uniaxial tension in the $x_{1}$ direction is simulated with the

322 Taylor and self-consistent models in conjunction with the Schmid and regularized Schmid 323 laws.

Figure 2 shows the macroscopic uniaxial stress-strain curve, where the flow stress is

326 normalized by critical resolved shear stress, $\tau_{0}$. For the Taylor model, the flow stresses predicted by both the Schmid and regularized Schmid laws are in good agreement. Since $N=500$ is large enough, the regularized Schmid law approaches Schmid one as is mentioned in section 2. The flow stresses, eventually, saturate to the value of 3.06 , which is the same as the well-known Taylor factor for rigid-plastic f.c.c. polycryatal.

332 For the self-consistent model, plastic yielding occur earlier than for the Taylor model and the

333 estimation of flow stress significantly depend on the crystal plasticity laws used in the 334 analysis. When the Schmid law is used, softer response is obtained. Indeed, the normalized 335 flow stress saturate to the value of 2.82. On the contrary, when the regularized Schmid law is 336 used, stiffer response is predicted and the stress state asymptotically approaches the one 337 predicted by the Taylor model. When the regularized Schmid law is used, the Taylor and 338 self-consistent models estimate almost the same behavior for the large strain range.

340 By definition, local strain states are the same as macroscopic one for the Taylor model and 341 vary for the self-consistent model. Figure 3 shows the evolution of local strain component 
342 normalized by the macroscopic tensile strain for the self-consistent model. The normalized

343 strain components for 216 phases are depicted. During the whole tensile straining process, the

344 Schmid law based self-consistent model predicts about $\pm 15 \%$ of constant variation of local

345 strain field. On the other hand, for the regularized Schmid law, similar variation is predicted

346 just after plastic yielding, but then the variation shrinks. At $\bar{\varepsilon}_{11}=0.05$, the variation is less

347 than $\pm 2 \%$. Thus, the self-consistent model with the regularized Schmid law is closer to the

348 Taylor model in terms of local field fluctuation. This small strain heterogeneity is consistent

349 with the macroscopic stress-strain curve that approaches the one of the Taylor model.

351 The local strain rate is specified by localization tensor in Equation (34), which includes the

352 local and effective tangent moduli and Hill's $\mathbf{P}$ tensor. The $\mathbf{P}$ tensor reflects the difference

353 of the local and effective tangent moduli to the localization tensor. The higher the components

354 of $\mathbf{P}$ are, the more enhanced the strain heterogeneity is. Components of $\mathbf{P}$ are shown in

355 Figure 4. For the Schmid model, the absolute value of the $\mathbf{P}$ components increase with

356 tensile straining. On the other hand, for the RSL, the $\mathbf{P}$ components are almost constant and

357 small. Therefore, the localization tensor is close to unit tensor and the fluctuation of local

358 strain field is small. The evolution of $\mathbf{P}$ tensor is in agreement with the local strain variation

359 in Figure 3.

361 As is shown in the equations (35) and (36), the $\mathbf{P}$ tensor depends on the effective tangent 362 moduli. Figure 5 shows the estimated effective tangent moduli in the rate-form of the 363 constitutive equations. The tangent moduli, $\tilde{L}_{i j k l}$, are normalized by the elastic moduli, $C_{i j k l}^{\mathrm{e}}$. 364 For the uniaxial tensile loading, the macroscopic tangent moduli display transverse isotropy

365 with respect to the $x_{1}$ direction, that is, $\tilde{L}_{2222}=\tilde{L}_{3333}, \tilde{L}_{1122}=\tilde{L}_{1133}$ and $\tilde{L}_{1212}=\tilde{L}_{1313}$. The 366 tangent moduli predicted by the two crystal plasticity laws do not agree except for 1111 and 3671122 components. Both tangent moduli depicted in Figure 5 correspond to uniaxial tension 368 state although they are not identical. The most significant difference is the shear tangent 369 moduli: those obtained with the Schmid law rapidly decrease after plastic yielding and 370 eventually reach to zero, whereas those for the regularized Schmid law are almost constant 371 during deformation. This trend is the same as that for the simple double slip case depicted in 
372 Figure 1(b). General relationships between $\mathbf{P}$ and $\tilde{\mathbf{L}}$ are given in Equations (35) and (36)

373 and analytical expressions can be found by Hutchinson (1970) for transverse isotropic

374 symmetry. $\mathbf{P}$ tensor increases when $\tilde{\mathbf{L}}$ decreases, thus the lower $\tilde{\mathbf{L}}$ components

375 pronounce a deviation of the localization tensor from the identity tensor.

377 For the Taylor model, SSL and RSL give the identical behavior so long as the exponent is 378 high enough. However, for the self-consistent model, the description of the tangent moduli 379 affects the local strain heterogeneity as well as effective response.

\subsection{Polycrystalline aggregate with an elastic phase}

In this subsection, the polycrystal aggregate is assumed to consist of 216 elastoplastic phases and an elastic phase. The elastic phase is considered in order to increase the contrast of the mechanical properties among phases. The volume fraction of the elastic phase is 0.3 and that of the other 216 phases are 0.00324 . The material properties of the 216 elastoplastic phases are the same as ones in 4.1, and the same elastic properties are assigned to an elastic phase.

All phases have spherical shape. Uniaxial tension in the $x_{1}$-direction is analyzed by means of the self-consistent model. Taylor model is not used here because the uniform strain field assumption is far from the reality for this problem.

Figure 6 shows the normalized flow stress as a function of the macroscopic tensile strain. The

392 Schmid law based model predicts softer response than that for the regularized Schmid law.

393 The qualitative tendency is the same as in Figure 2, and the existence of an elastic phase 394 enhances the difference of effective response. Figure 7 shows the local strain distribution of 395 the 216 elastoplastic and the elastic phases. The strain variation among the elastoplastic 396 phases is larger for the Schmid law than the regularized Schmid law. For the Schmid law, the 397 strain evolution in an elastic phase decreases with tensile strain, and the strain is much smaller 398 than that for the elastoplastic phases. On the other hand, for the regularized Schmid law the 399 strain develops linearly and its amount is about half of the elastoplastic phases. The Schmid 400 law allows lager strain heterogeneity among elastoplastic phases as well as between 401 elastoplastic and elastic phases. Figure 8 shows the effective shear tangent moduli normalized 
402 by the same way as in 4.1. Again, the shear moduli decrease rapidly after yielding for the

403 Schmid law, whereas those for the regularized Schmid law are almost constant. The influence

404 of the tangent moduli emerges again for this problem and the strong contrast of the phases

405 enhances this effect on the local and effective response.

\section{Discussion}

408 The description of standard Schmid law and regularized Schmid law has been examined for a uniaxial tensile loading (Section 2). It has become clear that both laws predict the same mechanical behavior of single grain under given boundary conditions, provided that the exponent in the regularized Schmid law is high enough. But, it has to be recalled that the

412 tangent moduli given by the two laws are different, although the same response is predicted.

413 For the non-hardening polycrystalline aggregate, the two laws with the Taylor model lead to

414 the same mechanical response under uniaxial tension. This can be explained by the fact that,

415 by definition, each phase undergoes the same strain field with the Taylor assumption. Since

416 the SSL and RSL predict the same response for a phase under the same boundary conditions,

417 the same mechanical response is predicted for each phase and consequently, the same local

418 and effective responses are obtained for the polycrystal. ${ }^{2}$ On the other hand, the two crystal

419 plasticity laws lead to different estimates with the incremental self-consistent model. The

420 shear tangent moduli for the SSL are lower than those for the RSL and are used to obtain

421 strain localization tensors which specify the average strain field within a phase. Therefore,

422 with the self-consistent model, the average strain field of a given phase varies between the

423 SSL and RSL. Furthermore, the lower the tangent moduli are, the more the strain

424 heterogeneity is enhanced. As a consequence, the self-consistent model with the SSL leads to

425 the larger strain heterogeneity and softer overall response. On the other hand, the

426 self-consistent model with the RSL predicts a smaller strain heterogeneity and a flow stress

427 approaching to the upper bound. From this point of view, it can be concluded that the

428 self-consistent model based on the SSL gives a better estimation.

\footnotetext{
${ }^{2}$ Uniaxial tensile behavior is also computed for the static model, which assumes a uniform stress field within the polycrystal. SSL and RSL with $N=500$ estimate the same behavior and the macroscopic stress is stationary at $\bar{\sigma}_{11} / \tau_{0}=2$.
} 
430 There are some similarities in the constitutive equations for the RSL and a power law 431 viscoplasticity model: (i) the slip law in equation (19) and the one for viscoplascity both obey 432 a power law, (ii) the regularized Schmid law possesses a smooth yield locus and the 433 viscoplastic model presents a smooth equal potential locus in stress space. The power law 434 viscoplastic model can thus also be considered as a regularization of the Schmid law. 435 Numerous investigations have been performed on the self-consistent model in the viscoplastic 436 context (see, among others, Hutchinson, 1976; Masson et al., 2000; Nebozhyn et al., 2001) 437 and it has been shown that the effective yield stress predicted by the Hill's incremental model 438 in the rate-independent limit is very close to the Taylor bound. By noting that this effective 439 yield stress corresponds to the saturated flow stress of a non-hardening elastoplastic 440 polycrystal, it is pointed out that our self-consistent estimates with a RSL are fully consistent 441 with the results obtained in the viscoplastic context. Following these remarks, it is likely that 442 any regularization (i.e. single yield function with associated flow rule) would lead to 443 incremental self-consistent estimates with the same shortcomings than the one reported in the 444 present study.

446 The physical interpretation of the reduced shear tangent moduli is considered here. First, a 447 situation is assumed such that the double slip single grain shown in Figure 1(a) is 448 continuously subjected to the uniaxial tension, and instantly shear strain rate, $\dot{\varepsilon}_{12}$, is applied 449 additionally. Schematic illustration of yield surfaces for the RSL and SSL in $\sigma_{11}-\sigma_{12}$ space 450 are shown in Figure 9. In these circumstances, the stress state is uniaxial tension, i.e. $\sigma_{i j}=0$ 451 except for $\sigma_{11}$, and non-zero strain rate components are $\dot{\varepsilon}_{11}, \dot{\varepsilon}_{22}$ and $\dot{\varepsilon}_{12}$. For the RSL, the 452 direction of the plastic strain rate is normal to the yield surface at the current stress state and $453 \quad \dot{\varepsilon}_{12}^{\text {p }}$ is not produced, hence $\dot{\varepsilon}_{12}$ is accomplished by elastic strain rate, $\dot{\varepsilon}_{12}^{\text {e }}$. Thus, the tangent 454 modulus of $L_{1212}$ is identical to the elastic shear modulus. On the other hand, for the RSL, 455 the direction of plastic strain rate lies between the normal directions of the facets. Therefore, 456 when $\dot{\varepsilon}_{12}$ is applied, $\dot{\varepsilon}_{12}^{\mathrm{p}}$ is produced and the tangent modulus is consequently reduced. The 457 introduction of the regularization, which avoids the difficulty of the selection of active slip 458 systems, loses the vertex effect on the tangent moduli. 
460 It is interesting to note an analogy between the standard and regularized Schmid laws and the 461 phenomenological $J_{2}$ flow and $J_{2}$ deformation theories. Reduced tangent moduli were 462 observed by Hutchinson (1970) who compared tangent moduli for the $J_{2}$ flow and 463 deformation theories. The flow theory assumes a smooth yield locus and associated flow rule 464 and the deformation theory reflects the point vertex effect on the yield surface. The flow and 465 deformation theories predict respectively constant and decreasing shear tangent moduli during 466 plastic deformation. The flow and deformation theories are similar to the RSL and SSL, 467 respectively. In fact, there is a vertex on the yield locus for the SSL and a single smooth yield 468 locus and associated flow rule are assumed in the RSL (Equations (16) and (18)).

In the crystalline context, our results highlight the fact that the flow stress estimate obtained with a regularized crystal plasticity law can be different from the one that is obtained with the non-smooth multi-criteria yield function corresponding to the SSL. In particular, the incremental self-consistent model is significantly affected by the mathematical description of the constitutive model and does not always lead to a stiff overall response with respect to the Taylor model. Indeed, with the incremental self-consistent model, the Schmid law gives a saturated flow stress of 2.82, which is lower than the estimation derived from the viscoplastic power law in the rate-independent limit $(\approx 3.06)$. Interestingly, it is noted that the saturated flow stress obtained with the SSL does not violate the variational self-consistent estimate of Ponte Castañeda for viscoplastic behavior which is equal to 2.948 in the rate-independent limit (Nebozhyn et al. 2001). To give an insight on the pertinence of Hill's incremental model

481 in the framework of the standard Schmid law, it would be necessary to compare the 482 self-consistent estimate of the saturated flow stress with more rigorous bounds: the one 483 derived for polycrystalline materials exhibiting a rigid-plastic behavior with a multi-criteria 484 local yield function (see, for instance, Nesi et al., 2000) or the recent improvement of the 485 variational approach of Ponte Castañeda (Idiart and Ponte Castañeda 2007a,b) which was 486 shown to improve on earlier bounds in the case of a two-phase porous crystalline material.

488 Concerning the RSL, it is interesting to note that different studies on particulate composites 
489 (matrix-inclusion microstructure) (Gonzales and LLorca, 2000; Doghri and Ouaar, 2003;

490 Chaboche et al., 2005) with an elastoplastic matrix obeying the $J_{2}$ flow theory have shown

491 that the incremental model deliver a too stiff overall stress-strain response by comparison with

492 finite element computations. This is qualitatively in agreement with our results obtained by

493 RSL for non-hardening polycrystal with an elastic phase. To obtain better self-consistent

494 estimates, it would be necessary to incorporate the field heterogeneity in the nonlinear

495 homogenization procedure for elastoplasticity.

\section{Concluding remarks}

498 The mechanical response of polycrystals has been investigated using the Hill's incremental 499 self-consistent model in conjunction with different rate-independent crystal plasticity laws. 500 The investigation of standard and regularized Schmid laws has revealed that they deliver the 501 same plastic deformation behavior but different tangent moduli. The use of these two 502 constitutive laws to describe the elastoplastic behavior of a non-hardening f.c.c. polycrystal 503 has shown that the standard Schmid law predicts a wider heterogeneity of the local strain field 504 and a softer overall response. We found that the reduced shear tangent moduli are responsible 505 for this behavior. The incorporation of an elastic phase in the elastoplastic polycrystal allowed 506 us to point out that an increase of the mechanical contrast implies a more pronounced 507 influence of the tangent moduli on the self-consistent description. The shear tangent moduli 508 decrease with the deformation for the SSL and remain constant for the RSL. With the 509 incremental self-consistent model, the Schmid law gives a saturated flow stress which is 510 lower than the estimation derived from the viscoplastic power law in the rate-independent 511 limit. The saturated flow stress obtained with the SSL (Hutchinson, 1970) does not violate the

512 variational self-consistent estimate of Ponte Castañeda for viscoplastic behavior in the 513 rate-independent limit. Comparisons with more restrictive bounds are thus required.

\section{7. Acknowledgements}

516 The authors acknowledge Nippon Steel Corporation for a financial support to this study. 


\section{References}

519 Anand, L., and Kothari, M., 1996. A computational procedure for rate-independent crystal 520 plasticity. Journal of the Mechanics and Physics of Solids, 44(4), 525-558.

521 Asaro, R., and Needleman, A., 1985, Texture development and strain hardening in rate 522 dependent polycrystals. Acta Metallurgica 33(6), 923-953.

523 Bassani, J.L., 1994. Plastic flow of crystals. In: Advances in Applied Mechanics, Volume 30.

524 Academic Press, London, pp. 191-258.

525 Brenner, R., Lebensohn, R. A., Castelnau O., Elastic anisotropy and yield surface estimates of 526 polycrystals, submitted.

527 Budiansky, B., Hashin, Z. and Sanders, J. L., 1960. The stress field of a slipped crystal and the 528 early plastic behavior of polycrystalline materials Plasticity. In: Proceedings of the 2nd Symp Naval Struct Mech., Pergamon, Oxford, 1960, 239.

Busso, E. P. and Cailletaud, G., 2005. On the selection of active slip systems in crystal plasticity. Internatinal Journal of Plasticity 21(11), 2212-2231.

Castelnau, O., Brenner, R. and Lebensohn, R.A., 2006. The effect of strain heterogeneity on the work hardening of polycrystals predicted by mean-field approaches. Acta Materialia, 54(10) 2745-2756.

Chaboche, J.L., Kanoute, P., Roos A., 2005. On the capabilities of mean-field approaches for the description of plasticity in metal matrix composites. International Journal of Plasticity 21 (7) 1409-1434.

Darrieulat, M., Piot, D., 1996. A method of generating analytical yield surfaces of crystalline materials. International Journal of Plasticity 12(5) 575-610.

Doghri, I., Ouaar, A., 2003. Homogenization of two-phase elasto-plastic composite materials and structures, Study of tangent operators, cyclic plasticity and numerical algorithms. International Journal of Solids and Structures 40 (7) 1681-1712.

Franciosi, P. and Berbenni, S., 2007. Heterogeneous crystal and poly-crystal plasticity

546 Franciosi, P. and Zaoui A., 1991. Crystal hardening and the issue of uniqueness. International $547 \quad$ Journal of Plasticity, 7(4) 295-311. 
548 Franz, G., Abed-Meraim, F., Lorrain, J.-P., Ben Zineb T., Lemoine, X. and Berveiller, M., 549 2009. Ellipticity loss analysis for tangent moduli deduced from a large strain $550 \quad$ elastic-plastic self-consistent model, International Journal of Plasticity, 25(2) 205-238.

551

552

553

554

555

556

557

558

559

560

561

562

563

564

565

566

567

568

569

570

571

572

573

574

575

576

577

Gambin, W., 1992. Refined analysis of elastic-plastic crystals, International Journal of Solids and Structures 29 (16) 2013-2021.

Gambin, W., Barlat, F., 1997. Modeling of deformation texture development based on rate independent crystal plasticity. International Journal of Plasticity 13(1-2) 75-85.

Gonzalez, C., LLorca, J., 2000. A self-consistent approach to the elasto-plastic behaviour of two-phase materials including damage. Journal of the Mechanics and Physics of Solids 48 (4) 675-692.

Hershey, A.V., 1954. The elasticity of an isotropic aggregate of anisotropic cubic crystals, Journal of Applied Mechanics, 21(9) 236-240

Hill, R., 1965. Continuum micro-mechanics of elastoplastic polycrystals. Journal of the Mechanics and Physics of Solids, 13(2) 89-101.

Hill, R. 1966. Generalized constitutive relations for incremental deformation of metal crystals by multislip. Journal of the Mechanics and Physics of Solids, 14(2), 95-102.

Hutchinson, J.W., 1970. Elastic-plastic behaviour of polycrystalline metals and composites. Proceedings of the Royal Society of London A 319(1537) 247-272.

Hutchinson, J.W., 1976. Bounds and self-consistent estimates for creep of polycrystalline materials. Proceedings of the Royal Society of London A 348(1652) 101-127.

Idiart, M.I. and Ponte Castañeda, P., 2007. Variational linear comparison bounds for nonlinear composites with anisotropic phases. I. General results. Proceedings of the Royal Society A, 463(2080), 907-924.

Idiart, M.I. and Ponte Castañeda, P., 2007. Variational linear comparison bounds for nonlinear composites with anisotropic phases. II. Crystalline materials. Proceedings of the Royal Society A, 463(2080), 925-943.

Kowalczyk, K. and Gambin, W., 2004. Model of plastic anisotropy evolution with texture-dependent yield surface Int. J. Plasticity, 20(1), 19-54.

Kröner, E., 1958, Berechnung der elastischen Konstanten des Vielkristalls aus den Konstanten des Einkristalls Z. Physik, 151, 504-518. 
578 Kröner, E., 1961. Zur plastischen Verformung des Vielkristalls. Acta Metallurgica, 9(2) 155-191.

580 Kröner, E., 1978. Self-consistent scheme and graded disorder in polycrystal elasticity. Journal of Physics F: Metal Physics, 8(11), 2261-2267.

Lebensohn, R.A., Liu, Y. and Ponte Castañeda, P., 2004. On the accuracy of the self-consistent approximation for polycrystals: comparison with full-field numerical simulations, Acta Materialia, 52(18) 5347-5361.

Masson, R., Bornert, M., Suquet, P. and Zaoui, A., 2000. An affine formulation for the prediction of the effective properties of nonlinear composites and polycrystals. Journal of the Mechanics and Physics of Solids 48(6-7), 1203-1227.

Miehe, C. and Schröder, J., 2001. A comparative study of stress update algorithms for rate-independent and rate-dependent crystal plasticity. International Journal for Numerical Methods in Engineering 50(2)273-298.

Mura, T, 1982, Micromechanics of defects in solids. Martinus Nijhoff Publishers, The Hague.

Nebozhyn, M. V., Gilormini, P. and Ponte Castañeda, P., 2001. Variational self-consistent estimates for cubic viscoplastic polycrystals : the effects of grain anisotropy and shape. Journal of the Mechanics and Physics of Solids, 49(2), 313-340.

Nesi, V., Smyshlyaev, V.P. and Willis J.R., 2000. Improved bounds for the yield stress of a model polycrystalline material. ournal of the Mechanics and Physics of Solids 48(9), 1799-1825.

Ponte Castañeda, P., 1991. The effective mechanical properties of nonlinear isotropic composite. Journal of the Mechanics and Physics of Solids, 39(1), 45-71.

Takahashi, H., 1988. Predictions of plastic stress-strain relations of polycrystals based on the

\section{A1. Influence of exponent, $N$, in regularized Schmid law}

605 The exponent, $N$, in the regularized Schmid law has been taken to 500 for the all 606 computations in Section 4. In this section, $N$ is taken to 5 and 50 in order to examine its 607 influence on the microscopic and macroscopic mechanical responses, and the problems in 
608 Section 4.1 and 4.2 are simulated. Figure A1(a) shows the macroscopic uniaxial stress-strain 609 curve for the isotropic non-hardening crystalline aggregate. The saturated flow stresses 610 predicted with the Taylor and self-consistent models coincide with each other for the same $611 N$ and decrease with $N$. When $N=5$, the flow stresses predicted by the Taylor and 612 self-consistent models are softer than the one predicted by the self-consistent model with the 613 Schmid law (Figure 2), since the yield stress of a single crystal becomes lower with 614 decreasing the exponent. Thus, this prediction does not imply that the regularized Schmid law 615 with $N=5$ is capable of predicting the softer effective response with large strain 616 heterogeneity. The development of the phase average strain for $N=5$ is shown in Figure 617 A1(b). The phase average strains once have a variation in the range of $-20 \%$ to $+10 \%$ just 618 after the plastic yielding and then, the variation shrinks. The amount of strain heterogeneity is 619 the same magnitude as the one for $N=500$ in Figure 3(b). The strain heterogeneity for $620 N=50$, which is not shown in the present paper, is almost the same as that for $N=500$. 621 Therefore, it is concluded that the exponent, $N$, has little influence on the estimation of 622 mechanical response.

624 The microscopic and macroscopic deformation of polycrystals reinforced by an elastic 625 inclusion, which is the problem in Section 4.2, is also simulated for $N=5$ and 50 , and the 626 results are shown in Figure A2. Softer behavior is predicted for the case of $N=5$, since lower 627 exponent gives softer flow stress for a single crystal. Figure A2(b) shows the strain 628 distribution in the polycrystalline and elastic phases. The predicted strain heterogeneity is 629 almost the same as the one for $N=500$ shown in Figure 7(b). We again conclude that the 630 exponent, $N$, in the regularized Schmid law has almost no influence on the estimation of the 631 mechanical behavior. 


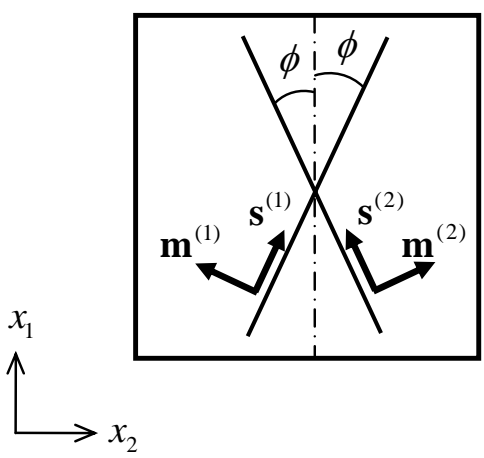

(a)

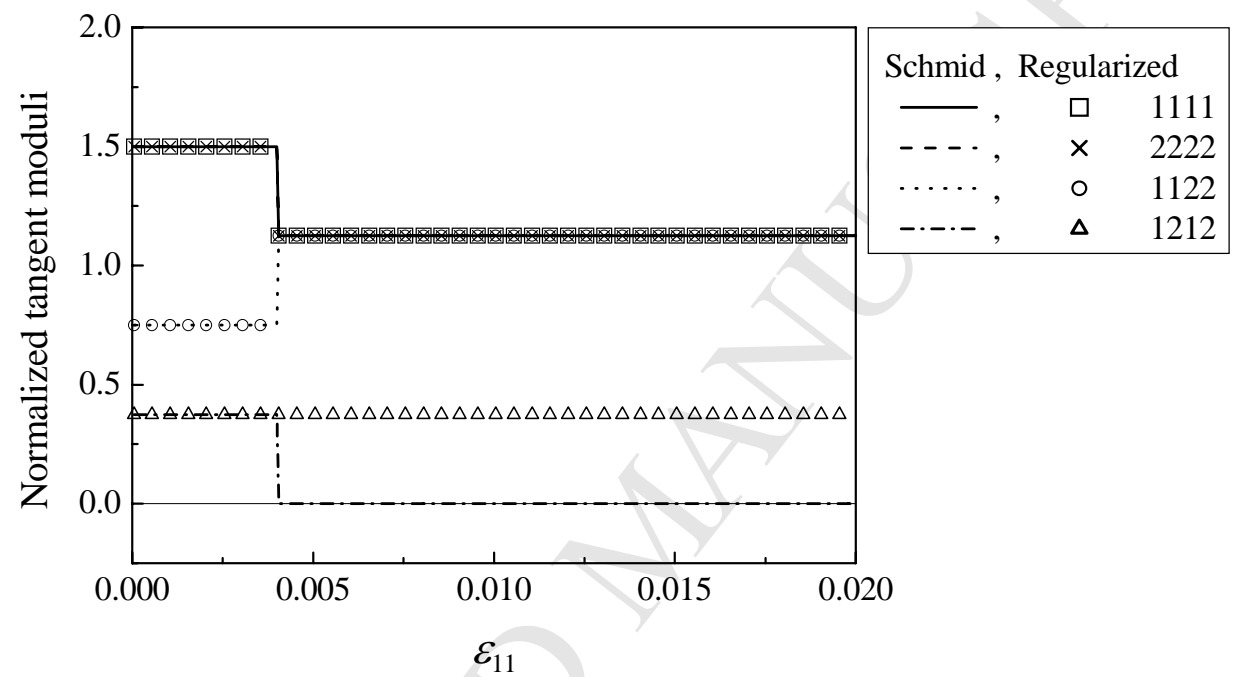

(b)

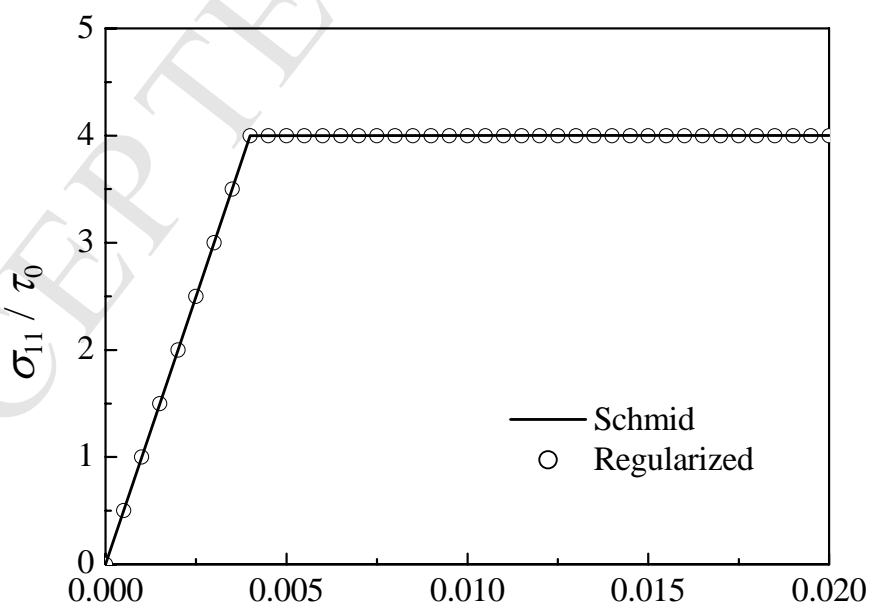

(c)

$\boldsymbol{E}_{11}$

4 Figure 1. Tangent moduli for a double slip crystal under uniaxial tension. (a) Geometry of

5 slip systems, (b) tangent moduli normalized by the Young's modulus and (c) tensile stress normalized by a critical resolved shear stress as a function of tensile strain. 


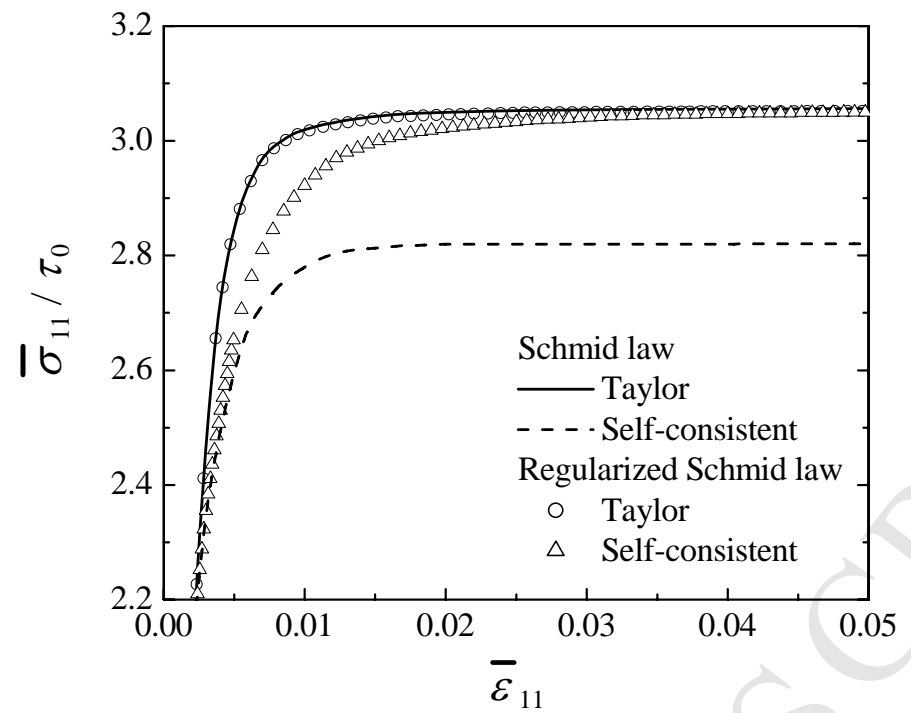

Figure 2. Macroscopic uniaxial stress-strain curve for isotropic non-hardening f.c.c.

9 crystalline aggregate. Taylor and incremental self-consistent models are used in conjunction 


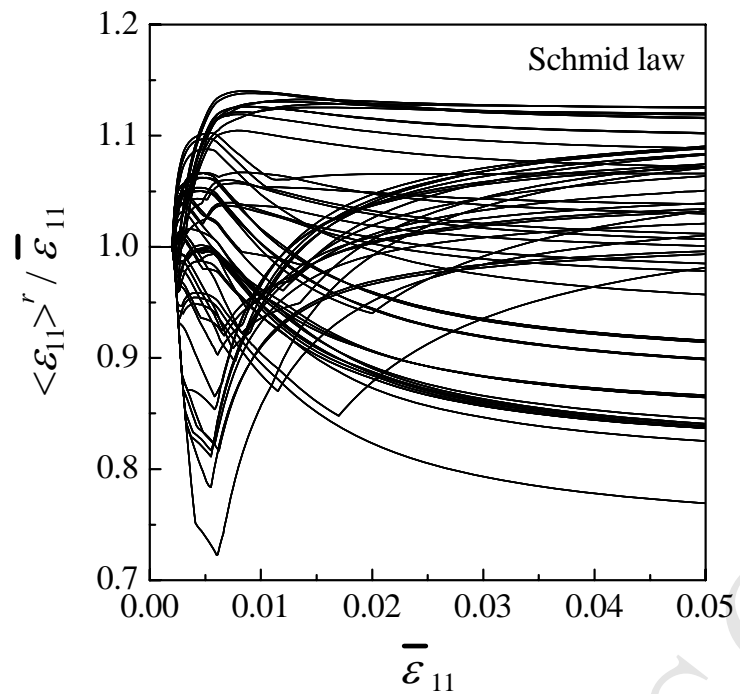

(a)

13

14

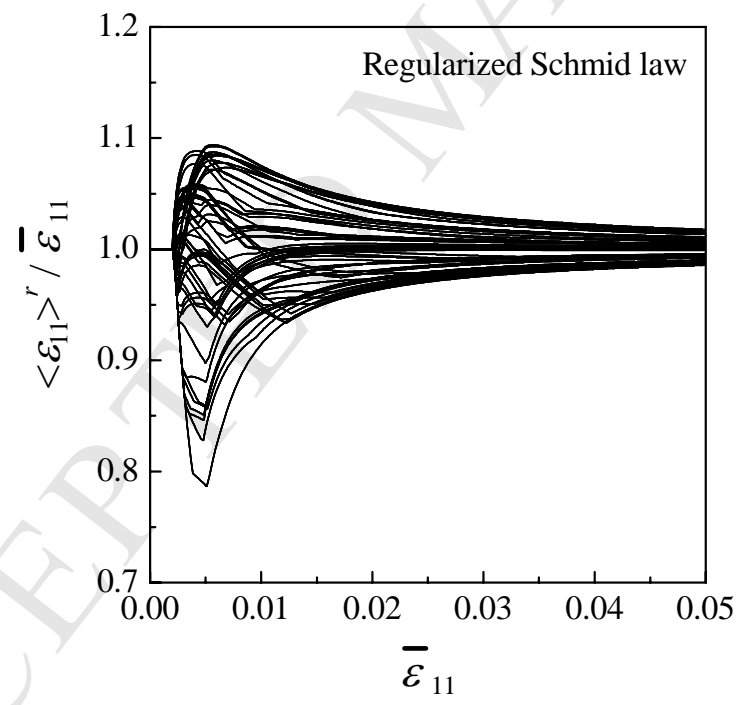

(b)

17 Figure 3. Evolution of local strain component, $\left\langle\varepsilon_{11}>^{r}\right.$, in 216 phases under uniaxial

18 tension. Incremental self-consistent model is used in conjunction with (a) Schmid law and (b) regularized Schmid law. 


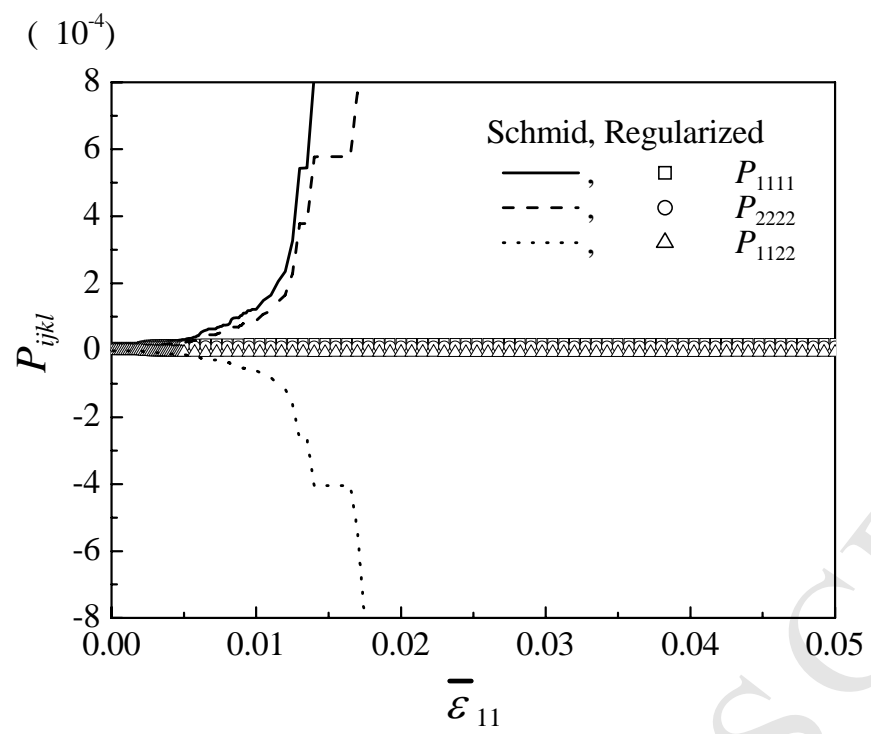

(a)

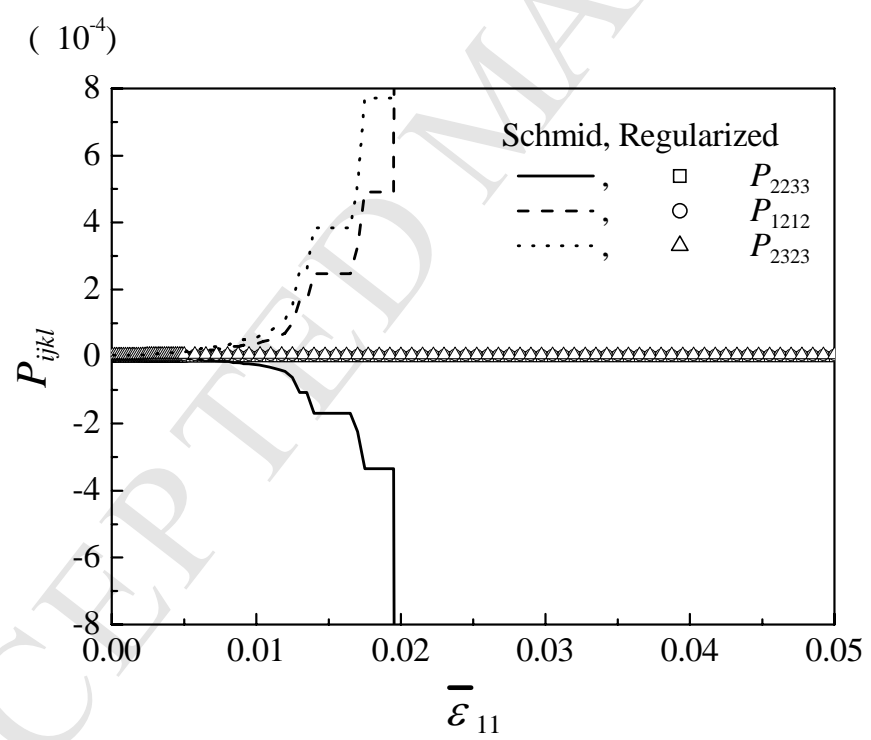

(b)

26 Figure 4. Evolution of $\mathbf{P}$ tensor for uniaxial tension as a function of macroscopic tensile 

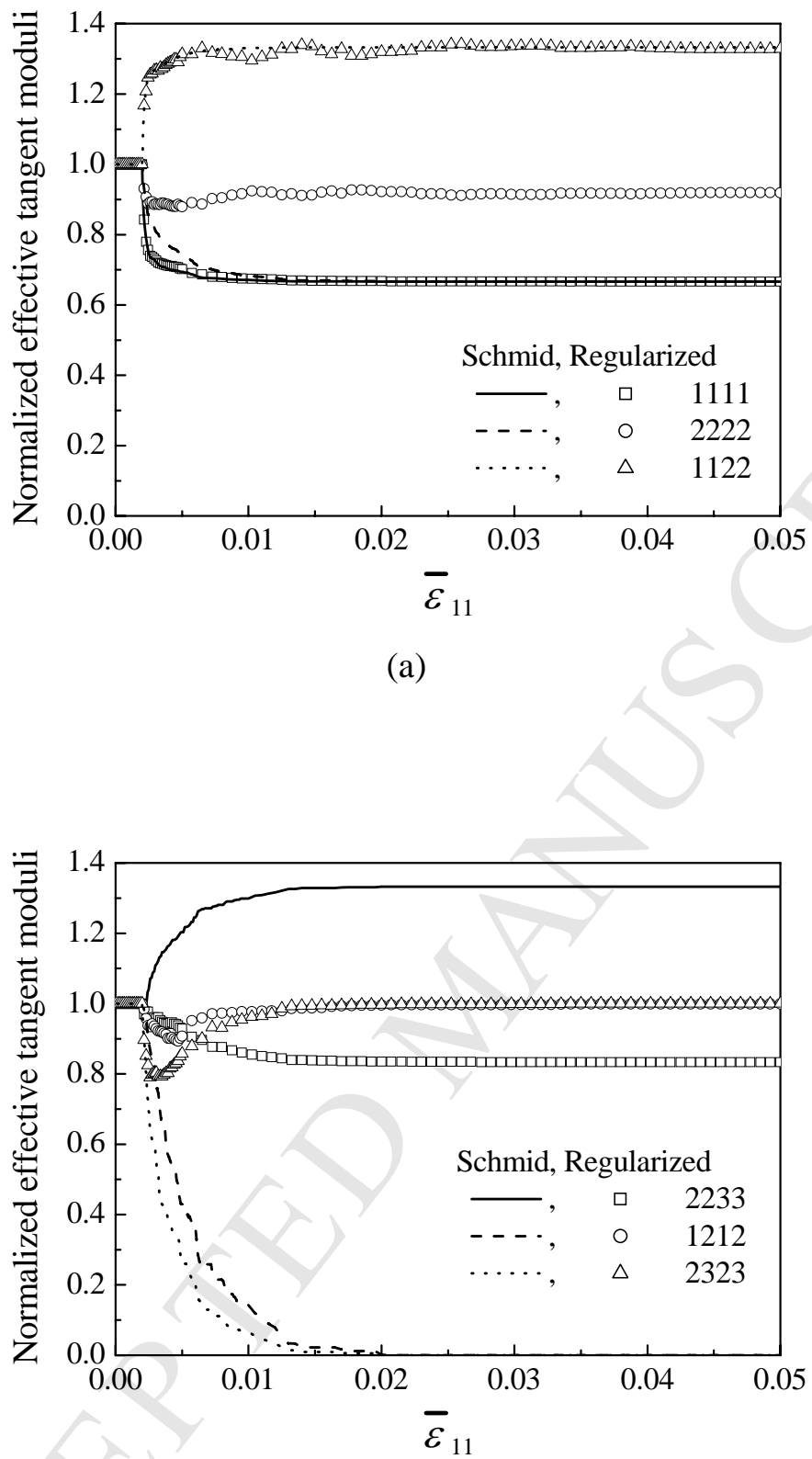

34 Figure 5. Evolution of tangent moduli under uniaxial tension. Effective tangent moduli, 


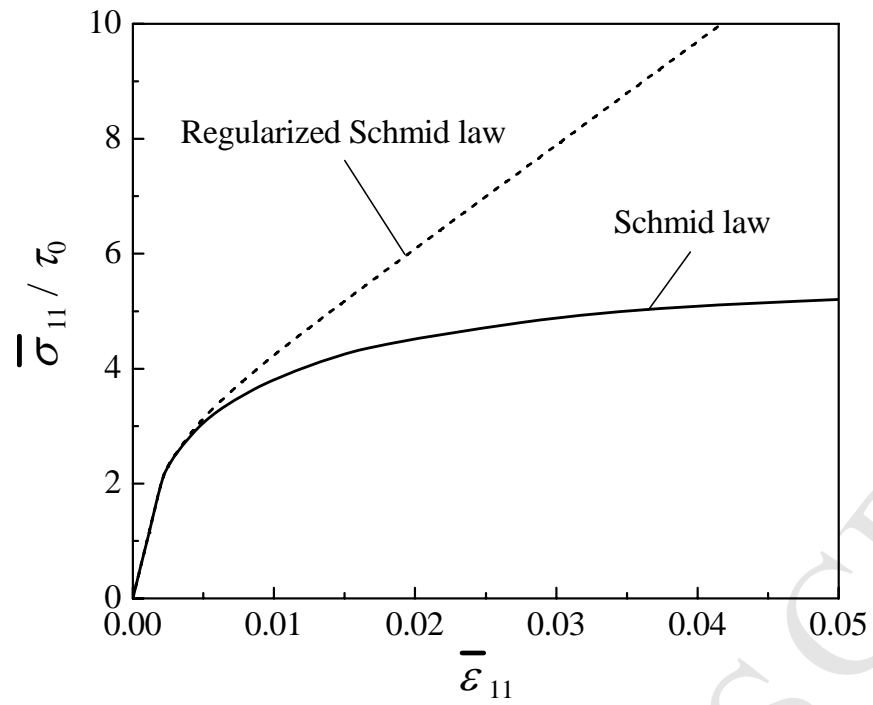

$37 \quad$ Figure 6. Stress-strain curve for aggregate consisting of elastoplastic phases and an elastic

38 phase. Incremental self-consistent model is used in conjunction with Schmid and regularized 


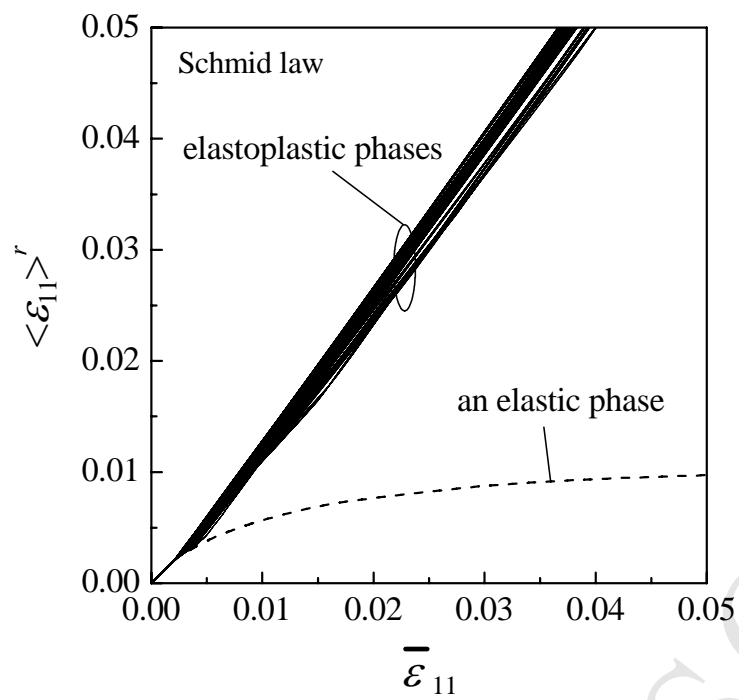

(a)

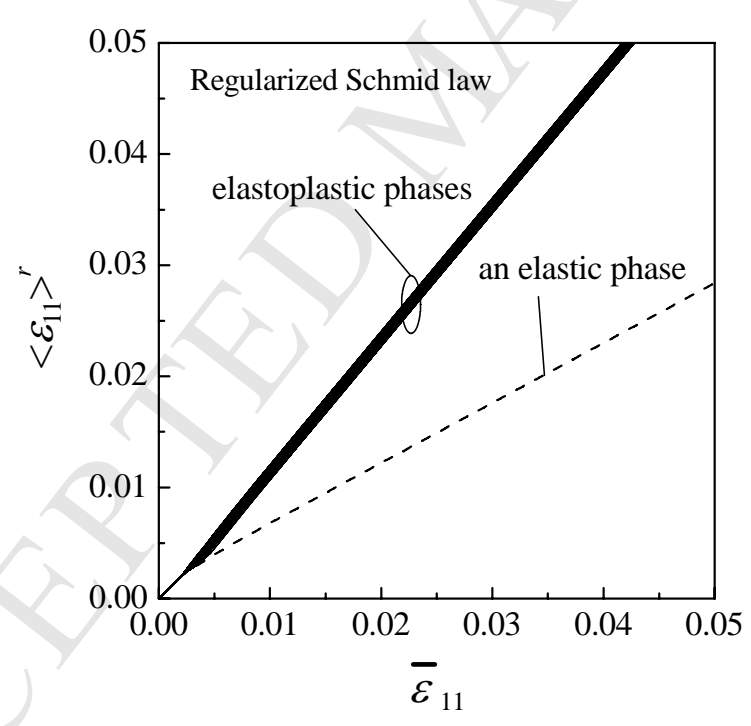

(b)

46 Figure 7. Evolution of local strain component, $\left\langle\varepsilon_{11}>^{r}\right.$, in 216 elastoplastic phases and an 47 elastic phase under uniaxial tension. Incremental self-consistent model is used in conjunction with (a) Schmid law and (b) Regularized Schmid law. 


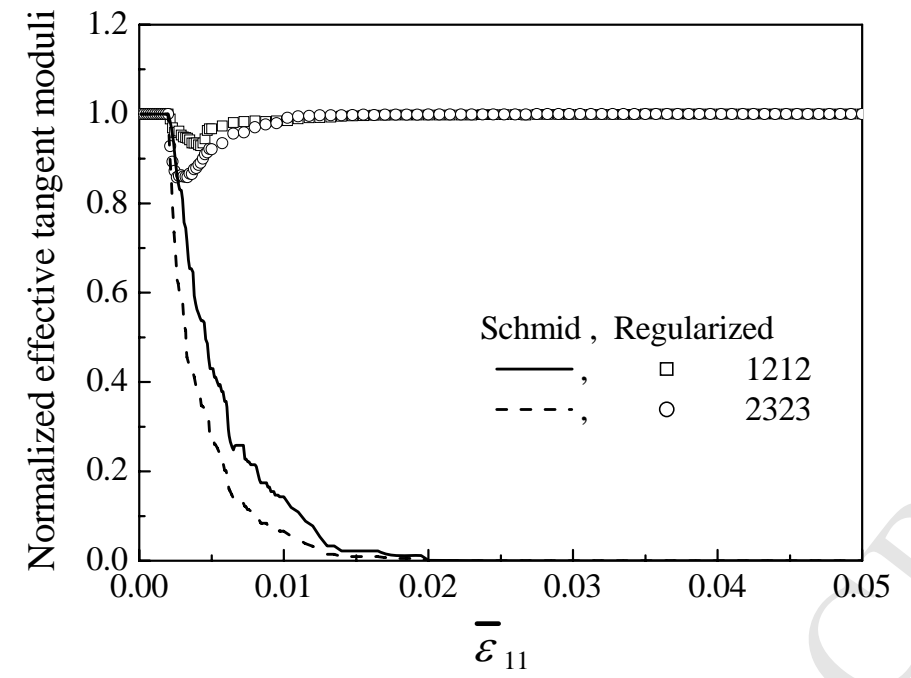

50 Figure 8. Evolution of tangent moduli for aggregate consisting of 216 elastoplastic phases 51 and an elastic phase under uniaxial tension. Effective tangent moduli, $\tilde{L}_{i j k l}$, are normalized by 


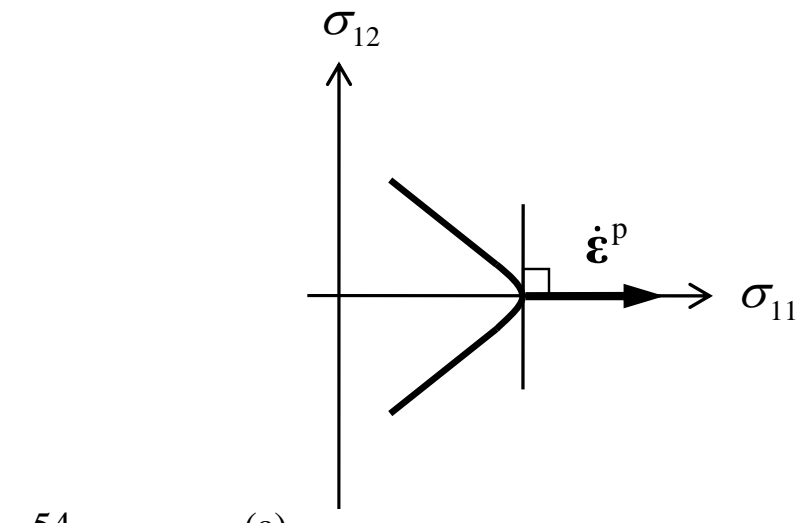

54

(a)

Figure 9 and (b) standard Schmid laws for a double slip crystal shown in Figure 1.

(b)

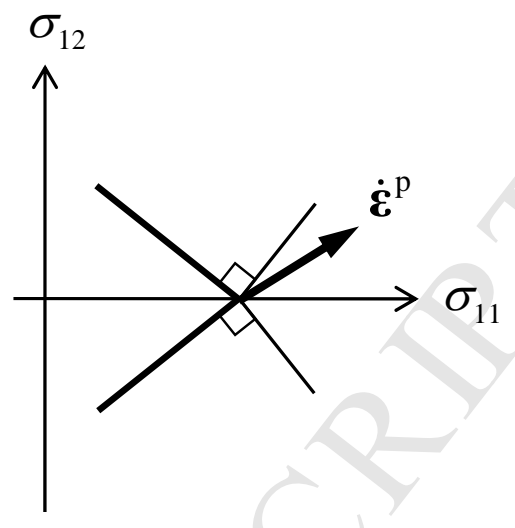

$\sigma_{11}-\sigma_{12}$ space based on (a) regularized 
(a)

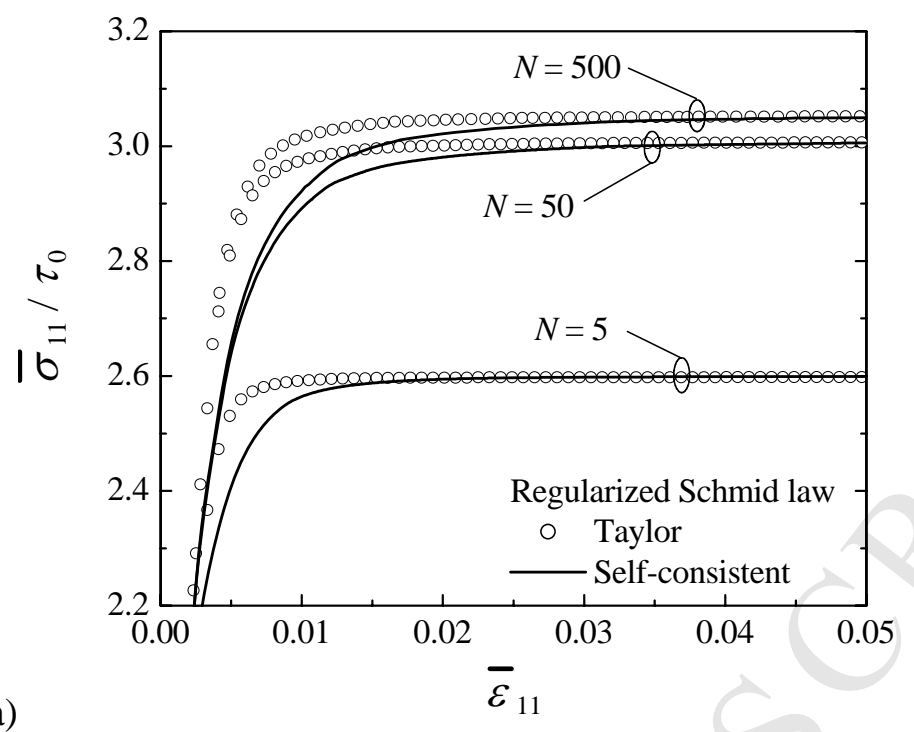

59

60

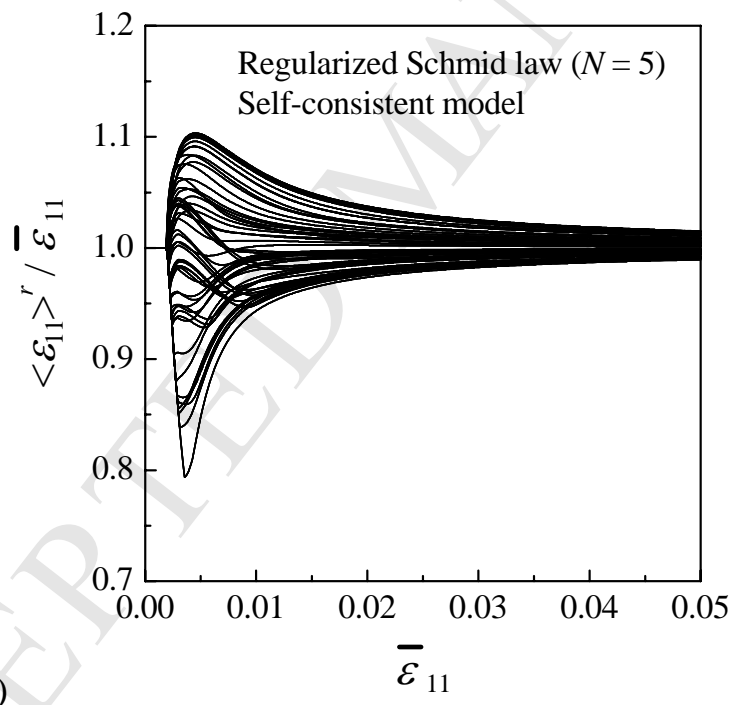

62 Figure A1. Influence of exponent in regularized Schmid law for isotropic non-hardening f.c.c. polycrystal. (a) Macroscopic flow stress and (b) strain heterogeneity. 


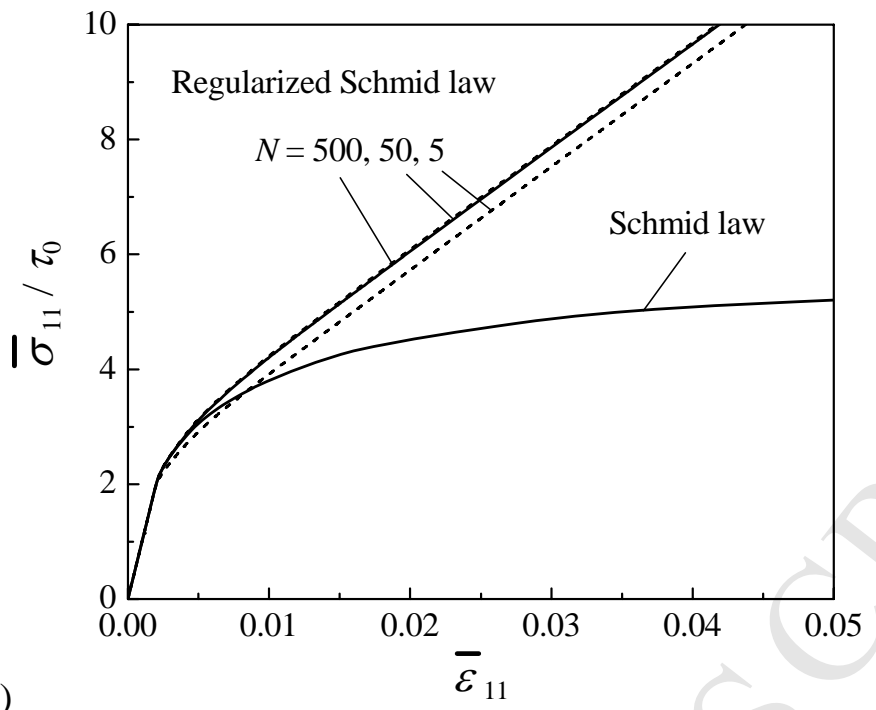

65

66

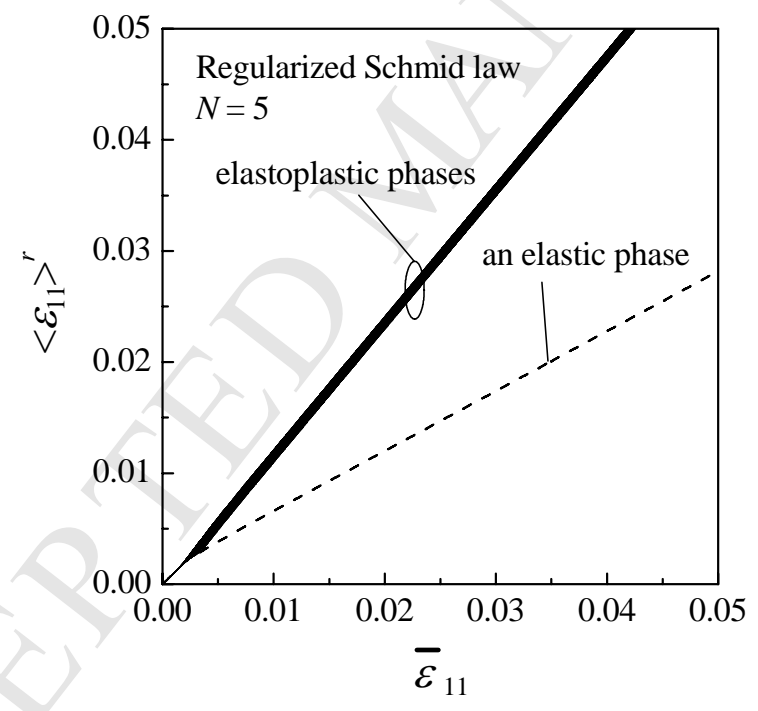

68 Figure A2. Influence of exponent in regularized Schmid law for isotropic non-hardening

69 f.c.c. polycrystal reinforced by an elastic inclusion. (a) Macroscopic flow stress and (b) strain 